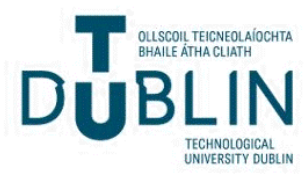

Technological University Dublin

ARROW@TU Dublin

\section{Antimicrobial Properties of Nano-silver: a Cautionary Approach to Ionic Interference}

\author{
Kate Sheehy \\ Technological University Dublin, kate.sheehy@tudublin.ie \\ Alan Casey \\ Technological University Dublin, alan.casey@tudublin.ie \\ Anna Murphy \\ Technological University Dublin, anna.murphy3@tudublin.ie
}

See next page for additional authors

Follow this and additional works at: https://arrow.tudublin.ie/scschphyart

Part of the Biological and Chemical Physics Commons

\section{Recommended Citation}

Sheehy, K., Casey, A., Murphy, A. \& Gordon Chambers (2015). Antimicrobial Properties of Nano-silver: a Cautionary Approach to Ionic Interference. Journal of Colloid and Interface Science, 443, pp. 56-64

This Article is brought to you for free and open access by the School of Physics \& Clinical \& Optometric Science at ARROW@TU Dublin. It has been accepted for inclusion in Articles by an authorized administrator of ARROW@TU

Dublin. For more information, please contact

arrow.admin@tudublin.ie, aisling.coyne@tudublin.ie, gerard.connolly@tudublin.ie.

Funder: Science Foundation Ireland, Fiosraigh DIT

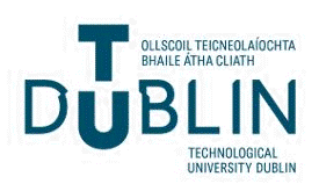




\section{Authors}

Kate Sheehy, Alan Casey, Anna Murphy, and Gordon Chambers

This article is available at ARROW@TU Dublin: https://arrow.tudublin.ie/scschphyart/75 


\title{
Antimicrobial properties of nano-silver: A cautionary approach to ionic interference
}

\author{
K. Sheehy*, A. Casey, A. Murphy, G. Chambers \\ Nanolab Research Group, School of Physics, Dublin Institute of Technology, Ireland
}

\section{A R T I C L E I N F O}

\section{Article history:}

Received 27 September 2014

Accepted 29 November 2014

Available online 6 December 2014

\section{Keywords:}

Nanotechnology

Antimicrobial

Disk diffusion

Nano-silver

\begin{abstract}
A B S T R A C T
Hypothesis: Metallic nanoparticles such as nano-silver have found many applications as alternative antimicrobials in recent years. However methods for determining their proposed antimicrobial activity have received little attention to date. The disk diffusion assay is commonly used as a demonstration of antimicrobial properties and is a regular feature in synthetic nanoparticle papers. The aim of this study was to assess its effectiveness in demonstrating the "nanoparticle specific" antimicrobial properties in the absence of ionic contributions from unreacted reducing agents and or impurities.

Experiments: The disk diffusion assay was carried out on a range of silver nanoparticles, both in-house synthesised and commercially available, using Escherichia coli ATCC 25922 as a model organism. Results: Capped and purified nanoparticles show no antimicrobial activity despite claims to the contrary for this assay. Results will be discussed in terms of the need for researchers without a background in microbiology to understand the mechanism of antimicrobial action before choosing an assay. Also discussed is the importance understanding the physiochemical characteristics of when interpreting results. Finally the relevance of the results in terms establishing protocols for method development for 'nanoparticle specific' antimicrobial properties will also be considered.
\end{abstract}

(c) 2014 Elsevier Inc. All rights reserved.

\section{Introduction}

The evolution of antibiotic resistant bacteria such as MRSA has resulted in a significant body of research focused on finding alternative antimicrobial agents capable of eradicating these superbugs [11]. One promising alternative which has been proposed is nanosilver [23]. Metallic nanoparticles in general have been reported to have broad spectrum activity against both Gram-positive and Gram-negative bacteria including antibiotic resistant bacteria like MRSA and MRSE $[2,18,20]$.

The mechanism of the antimicrobial properties of metallic nanoparticles is still under debate with the consensus being that it is due to ionic contributions rather the any "nanoparticle specific" activity [30]. Nevertheless there has been a significant upsurge in reports of nanomaterials exhibiting antimicrobial properties $[25,21,1,15,9]$. A deeper investigation reveals that many of these reports merely append a simplistic disk diffusion assay or a variation of this assay to essentially a material synthesis report $[26,10]$. Often such studies fail to take account of the physicochemical properties of the nanoparticle which have been crucially important in helping to standardise approaches for the assessment

\footnotetext{
* Corresponding author.

E-mail address: kate.sheehy@dit.ie (K. Sheehy).
}

of nanoparticle toxicity and are likewise expected to contribute significantly to understanding the antimicrobial properties of nanomaterials $[19,28]$. In addition, limited statistical analysis is often carried out in the aforementioned work with the inhibition zone being taken as conclusive evidence that the nanomaterial under study is antimicrobial with no regard to impurities, particle coatings, competing processes or known mechanisms of interaction $[25,1,17]$.

The choice of the disk diffusion assay in these circumstances is usually based on the fact that it is a relatively economical and easy assay to perform, in principle. Indeed it is a common method for testing the sensitivity of bacteria to antibiotics, which are soluble molecules with minimal impurities. In the disk diffusion assay a micro-organism is classed as sensitive or resistant to a material based on the radius of the zone of inhibition which forms around a small filter paper disk containing the test material. In comparison to antibiotics, the properties of nanomaterials are less consistent, with significant variations between batches in terms of particle size distribution, bioactivity, purity and surface chemistry $[28,19]$. Furthermore nano-metals such as nano-silver are often insoluble in aqueous solution and have a tendency to agglomerate in certain environments such as nutrient broth [19] making them unsuitable for many assays other than the disk diffusion assay. In addition nano-metals are also particularly efficient at forming 
metal ions in solution which are well known to exhibit antimicrobial properties [6]. Ion formation must be taken into account when selecting an assay and in the interpretation of subsequent results to distinguish the nano-particle specific effect from the ionic effect. This is particularly true when considering particles which have been stabilised in aqueous solvents (using coating/capping agents such as polyvinylpyrolidone (PVP), citrate buffers or surfactants) which will have a reduced capacity to form ions, and for 'as-produced' unpurified nanoparticles which will have unreacted reducing agents present from the synthesis [25]. Considering these aspects, a key question that this paper will address is whether or not the disk diffusion assay is a suitable method for assessing the antimicrobial nature of nano-metals. The paper will examine the most appropriate approach to differentiate between the particle and the ionic effect thereby helping to interpret the results of the disk diffusion assay for a range of nano-silver particles, coated and uncoated. It will also identify several pitfalls which researchers should be aware of with respect to the processing of nanomaterials prior to testing. The results reported reaffirm the need to characterise the physicochemical properties of the nanoparticle prior to assessing any potential antimicrobial properties analogous to the approach taken in the emerging area of nanotoxicology [28]. Finally the relevance of the results in terms establishing protocols for method development for 'nanoparticle specific' antimicrobial properties will be discussed.

\section{Materials and methods}

\subsection{Nanoparticles}

PVP-coated silver nanopowder, particle size $<100 \mathrm{~nm}$ was purchased from Sigma Aldrich, Ireland (product number 576832). A $20 \mathrm{ppm}$ stock solution of $20 \mathrm{~nm}$ citrate stabilisedzed nano-silver solution, particle size $<20 \mathrm{~nm}$ was purchased from Sigma Aldrich Ireland (product number 730793). The above nanoparticle products were used as standards, due to their high quality, monodispersity and purity. As well as these, a third set of uncoated particles were synthesised by a sodium citrate reduction of silver nitrate, as described by Fang et al. [8]. A stock $10^{-3} \mathrm{M} \mathrm{AgNO}_{3}$ aqueous solution (Sigma Aldrich, Ireland) and a stock 1\% trisodium citrate solution (Sigma Aldrich, Ireland) were prepared. $500 \mathrm{ml}$ of $\mathrm{AgNO}_{3}$ aqueous solution was heated to boiling, and to this solution $10 \mathrm{ml}$ of trisodium citrate solution was added drop by drop with vigorous magnetic stirring. Heating and stirring were continued for 30 min until a clear yellow liquid (silver nanoparticle solution) was observed in the flask. The nanoparticle presence was confirmed using a Perkin Elmer Lambda 900 UV/VIS/NIR Spectrometer to indentify the silver plasmon as discussed in previous studies $[7,26]$. These in-house synthesised and commercial nanoparticles were then characterised using multiple techniques detailed below to estimate particle size.

\subsection{Culture media}

Nutrient broth was purchased from Sigma Aldrich, Ireland (product number 70122). $25 \mathrm{~g}$ of nutrient broth was mixed with $1 \mathrm{~L}$ of deionised water before being autoclaved. Nutrient agar was prepared by adding $15 \mathrm{~g}$ of bacteriological agar (Sigma Aldrich product number A5306) to $25 \mathrm{~g}$ of nutrient broth before adding $1 \mathrm{~L}$ of deionised water and autoclaving.

\subsection{Bacterial strains}

The majority of researchers use either/or Escherichia coli and Staphylococcus aureus [25,31,21]. Therefore as an initial representative strain, E. coli ATTC strain 25,922 was chosen and purchased from ATCC. E. coli was taken from frozen stocks and subcultured onto nutrient agar before being incubated at $37^{\circ} \mathrm{C}$ for $24 \mathrm{~h}$. This was then subcultured twice more in the same manner before being used in experiments.

\subsection{Nanoparticle characterisation}

As an initial step in all nanoparticle work, it is crucial to perform a series of physiochemical characterisation steps to establish the nature of the particle under study [19,28]. Nanoparticles can aggregate over time and in different environments, forming larger species than may be expected (>100 $\mathrm{nm}$ ) thereby reducing "nanoparticlespecific" effects. As a result it has become good practice in nanomaterial laboratories to physically confirm specifications of particles prior to use [22]. While the type of characterisation required may vary for different particles and studies, it is expected that at a minimum the particle size range or distribution would be determined. All particles used in this study where characterised for size/distribution using two methods; Dynamic Light Scattering (DLS) and Scanning Electron Microscopy (SEM). In addition particle purity and coatings (where applicable) were confirmed using UV-Visible absorption spectroscopy [5], X-ray diffraction spectroscopy (XRD) [4], and atomic absorption spectroscopy (AAS) [13]. Colloidal stability was assessed using zeta potential analysis [27]. For the purpose of clarity for this paper only details of methods pertaining to particle size distribution and were appropriate purity or concentration will be presented and discussed.

\subsubsection{Particle size distribution}

Dynamic Light Scattering (DLS), also known as Photon Correlation Spectroscopy, or Quasi-Electrical Light Scattering, is a non invasive technique used to measure particle size distributions in solvated environments, typically in the nanometer size range. The parameter that is measured by Dynamic Light Scattering is the hydrodynamic radius, which refers to how a particle diffuses in a fluid [16]. Applications of DLS are typically the measurement of particles in a liquid, e.g. proteins, polymers, micelles, nanoparticles, colloidal suspensions, and emulsions [16,19].

DLS was used to establish particle size of both commercially available and in house synthesised nano-metal samples. A Malvern Nano series ZS Zetasizer, operating of version 7.10 of the DTS Nano Software, was employed for all analysis. All samples were made up to a $20 \mathrm{ppm}$ concentration in deionised, ultra-pure water and measured in triplicate.

\subsubsection{Particle stability}

Zeta potential is the measure of the stability of a colloid system. When particles are suspended in a media of different phase (e.g. solids suspended in liquid) their interaction with the media surrounding them determines whether the particles will coagulate or stay dispersed. In this case, the stability of the nanoparticles were measured both in water and in nutrient broth.

A Malvern Nano series ZS Zetasizer, operating of version 7.10 of the DTS Nano Software, was employed for all analysis. All samples were made up to a $20 \mathrm{ppm}$ concentration in deionised, ultra-pure water/nutrient broth and measured in triplicate.

\subsubsection{Particle size and shape}

Scanning Electron Microscopy (SEM) was used to determine particle size and shape. There have been reports associating nanoparticle shape with different biological responses and so it was deemed important to ensure that all particles in this study were approximately the same shape (for example spherical) [24]. For the SEM characterisation all nanoparticle samples were diluted in ethanol, spin coated onto a cleaned silicon wafer and imaged using 
a Hitachi SU6600 FESEM SE (Secondary Electron) Detector at $5 \mathrm{kV}$ $\& 10 \mathrm{kV}$ without coating. Multiple images were taken of each sample. The process was repeated in triplicate. Images were analysed using Image software [Branch 2008] to determine average size and shape.

\subsubsection{Concentration and purity determination}

To establish the concentration of the nanoparticle suspensions that were produced in-house, samples were analysed using a Varian SpectrAA-200 atomic absorption spectrometer, and their concentration determined using a standard curve.

\subsection{Purification of nanoparticles/ion separation}

In order to purify the in-house synthesised particles and remove any dissolved ions/impurities, nanoparticle sample was filtered using a $10 \mathrm{kDa}$ centrifugal filter unit by centrifuging at $2867 \times \mathrm{g}$ for $20 \mathrm{~min}$. The retentate was then re-suspended in ultrapure deionised water to the desired concentration and brought forward for testing. The supernatant was also kept for testing. This method was also carried out on the citrate stabilised Sigma Aldrich nanoparticle suspension and treated in the same manner. Both the retentate and the supernatant from all of the above samples were analysed using AAS as described above to determine particle concentration and presence of ionic impurities in the supernatant.

\subsection{The disk diffusion assay}

The disk diffusion assay was first described by Bauer et al. [3]. In order to carry out this assay with nanoparticles as opposed to traditional antibiotics the method was adapted slightly Shahaverdi et al. [26] have previously outlined a disk diffusion method suitable for the determination of the antimicrobial activity of nanoparticles [26]. A modified version of this method was performed here. In brief, organisms were prepared by culturing on nutrient agar overnight. Following incubation, a standard inoculum of each bacterial strain was prepared in sterile water to a concentration of $1.5 \times 10^{8} \mathrm{CFU} / \mathrm{ml}$ (0.5 McFarland Standard). A sterile swab was dipped into the suspension and then streaked onto a pre prepared nutrient agar plate in order to provide a uniform coverage of bacteria on the surface of the plate. For this experiment, all of the nanoparticle samples were tested in concentration ranges from 0 to $100 \mathrm{ppm}$. $40 \mu \mathrm{l}$ of the nanoparticle suspension was then aliquoted onto a sterile filter paper disk (purchased from Oxoid, Ireland). The disk was then placed on top of the surface of the plate. Numerous disks could be placed on the same plate. All disks and plates were labelled and incubated for $24 \mathrm{~h}$ at $37^{\circ} \mathrm{C}$.

\section{Results}

\subsection{Nanoparticle characterisation}

As aforementioned, the physicochemical characterisation of nanoparticles as exposed is a critical step in the interpretation of biological assays and the subsequent elucidation of any 'nanoparticle-specific' responses which may be identified. The particles in this study have been characterised via multiple techniques, details of the results relevant to this paper namely size distribution and shape are presented in Table 1 . The comparison to the manufacturer's specification has also been given.

From Table 1, it can be seen that all measurements were in agreement with the manufacturers specifications for the commercial particles. SEM analysis did reveal a degree of aggregation in the particles reflected by the larger mean in the particle size, which has been attributed to the spin coating process enhancing the potential for nanoparticle-nanoparticle interaction as the solvent is removed, nevertheless all particles remained below the notional $100 \mathrm{~nm}$ barrier for nanoparticles. The largest particles were identified in the citrate coated nano-silver were a range of $46 \pm 24 \mathrm{~nm}$ in size was observed. Colloidal stability studies also confirmed the aggregation of the particles in aqueous environment, being even more pronounced in the nutrient broth. In particular with respect to the uncoated in-house synthesised particles were clear aggregation was seen post $48 \mathrm{~h}$. This however was sufficient time to perform any sample preparation and exposure required for the disk diffusion assay (see Figs. 1 and 2).

\subsection{Disk diffusion assay on commercially purchased particles}

100 ppm stock solutions of both the citrate and PVP coated commercial particles were prepared in deionised ultra pure water and serially diluted for testing. $40 \mu \mathrm{l}$ of each subsequent nanoparticle suspension was then aliquoted onto the sterile filter paper disks ready for exposure. In all experiments ampicillin was employed as a positive control while sterile deionised water was used as the negative control. All experiments were carried out in triplicate. Figures below show typical results as seen in one experiment. Table 2 shows the inhibition zone diameters for all three sets of experiments, expressed as a mean value \pm standard deviation.

Fig. 5 shows the disk diffusion assay carried out on E. coli using the commercial citrate stabilised nano-silver dispersion over a concentration range of 10-100 ppm nano-silver. The assay displayed no inhibition zone around the disks containing the nano-silver or the negative control (Fig. 5a left lower quadrant). This suggests that the citrate stabilised nano-silver at concentrations up to $100 \mathrm{ppm}$ has no observable antimicrobial effect on $E$. coli. The positive control, ampicillin did display a zone of inhibition of approximately $0.6 \mathrm{~cm}$ (Fig. 5a right lower quadrant).

Table 1

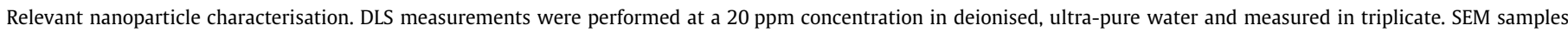
were spin coated onto silicon from ethanol and analysed in Image J software. Data is expressed as the mean \pm standard deviation.

\begin{tabular}{|c|c|c|c|c|c|c|c|}
\hline \multirow[t]{2}{*}{ Material } & \multicolumn{3}{|c|}{ Particle size (nm) } & \multicolumn{2}{|l|}{ Particle shape } & \multicolumn{2}{|c|}{ Zeta potential $(\mathrm{mV})$} \\
\hline & $\begin{array}{l}\text { Manufacturer's } \\
\text { specifications }\end{array}$ & DLS & SEM & $\begin{array}{l}\text { Manufacturer's } \\
\text { specifications }\end{array}$ & SEM & In water & $\begin{array}{l}\text { In nutrient } \\
\text { broth }\end{array}$ \\
\hline $\begin{array}{l}\text { Sigma Aldrich }{ }^{\mathrm{TM}} \text { citrate ctabilised nano- } \\
\text { silver dispersion }\end{array}$ & $20 \pm 4$ & $21.04 \pm 2.3$ & $46 \pm 24$ & Spherical & Spherical & $-25.23 \pm 5.3$ & $-7.53 \pm 1.0$ \\
\hline $\begin{array}{l}\text { Sigma Aldrich }{ }^{\mathrm{TM}} \text { PVP-stabilised nano-silver } \\
\text { powder }\end{array}$ & $<100$ & $30.8 \pm 0.94$ & $50 \pm 10$ & Spherical & Spherical & $-31.2 \pm 0.5$ & $-8.51 \pm 3.0$ \\
\hline In house synthesised (impure) & $\mathrm{n} / \mathrm{a}$ & $12.99 \pm 1.0$ & $40 \pm 10$ & $\mathrm{n} / \mathrm{a}$ & Spherical & $-37.26 \pm 1.4$ & $-15.66 \pm 0.7$ \\
\hline $\begin{array}{l}\text { In-house synthesised (pure)-concentrated } \\
\text { stock }\end{array}$ & $\mathrm{n} / \mathrm{a}$ & $16.62 \pm 1.6$ & $40 \pm 10$ & $\mathrm{n} / \mathrm{a}$ & Spherical & $-34.93 \pm 0.9$ & $-7.9 \pm 5.2$ \\
\hline
\end{tabular}




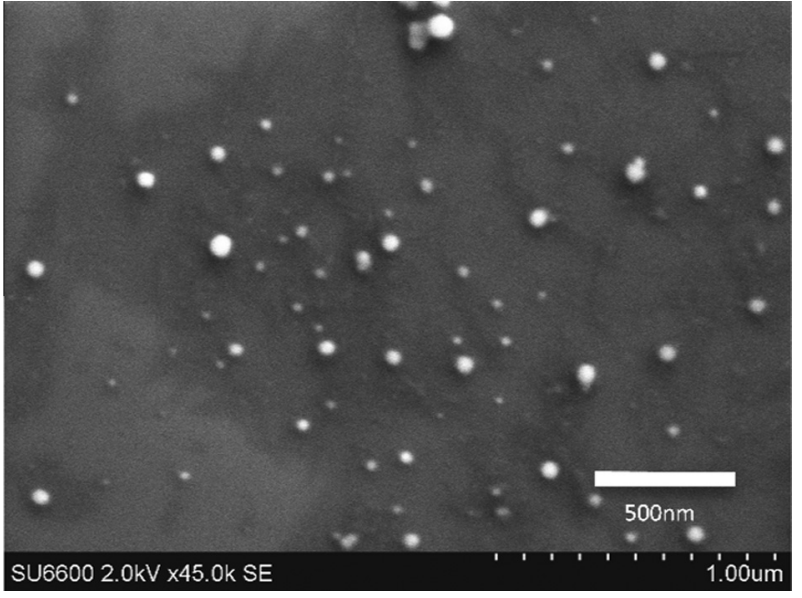

Fig. 1. SEM image at scale bar of $1 \mu \mathrm{m}$ showing citrate stabilised silver nanoparticles as purchased dispersed in ethanol to a concentration of $10 \mathrm{ppm}$ by sonication using Ultrasonic Processor. SEM images of various silver nanoparticles, all prepared by dispersion in ethanol followed by sonication. Samples were spin coated onto silicon wafers before being analysed in the SEM.

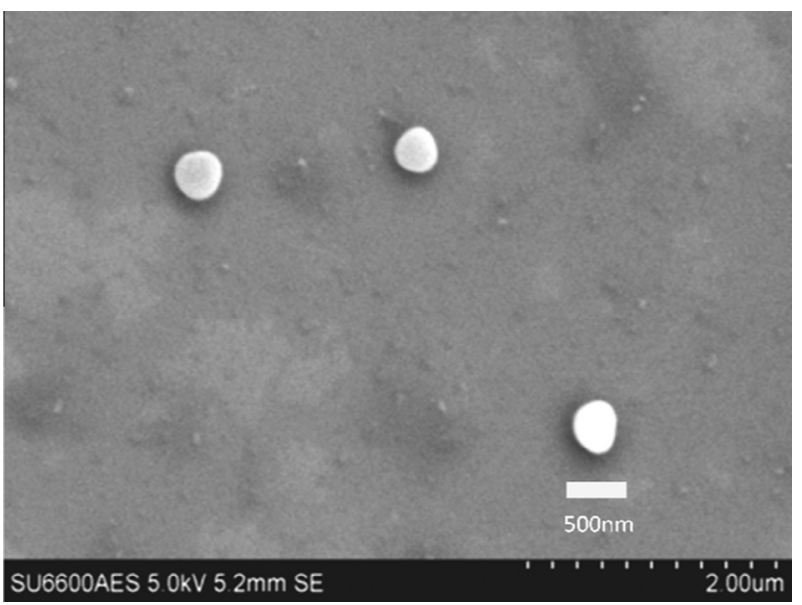

Fig. 2. SEM image at scale bar of $2 \mu \mathrm{m}$ showing PVP stabilised silver nanoparticles as purchased dispersed in ethanol to a concentration of $10 \mathrm{ppm}$ by sonication using Ultrasonic Processor tip. SEM images of various silver nanoparticles, all prepared by dispersion in ethanol followed by sonication. Samples were spin coated onto silicon wafers before being analysed in the SEM.

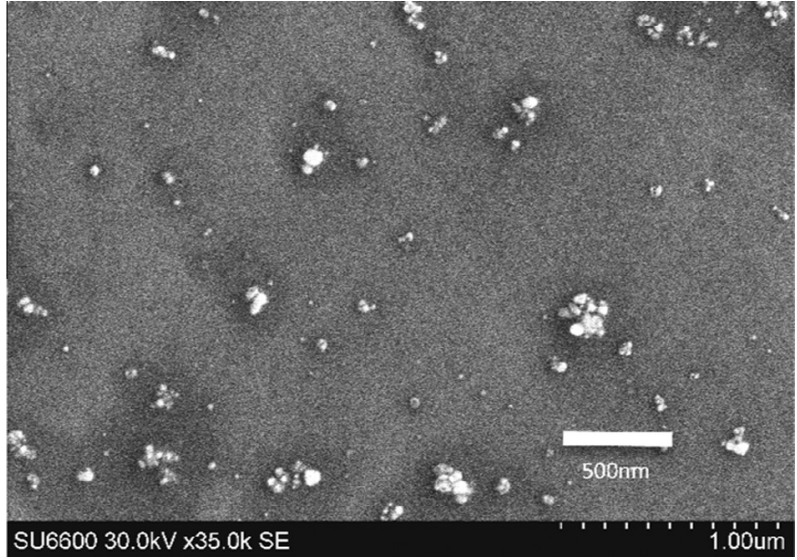

Fig. 3. SEM image at scale bar of $1 \mu \mathrm{m}$ showing impure in-house synthesised silver nanoparticles as purchased dispersed in ethanol to a concentration of $10 \mathrm{ppm}$ by sonication using Ultrasonic Processor tip. SEM images of various silver nanoparticles, all prepared by dispersion in ethanol followed by sonication. Samples were spin coated onto silicon wafers before being analysed in the SEM.

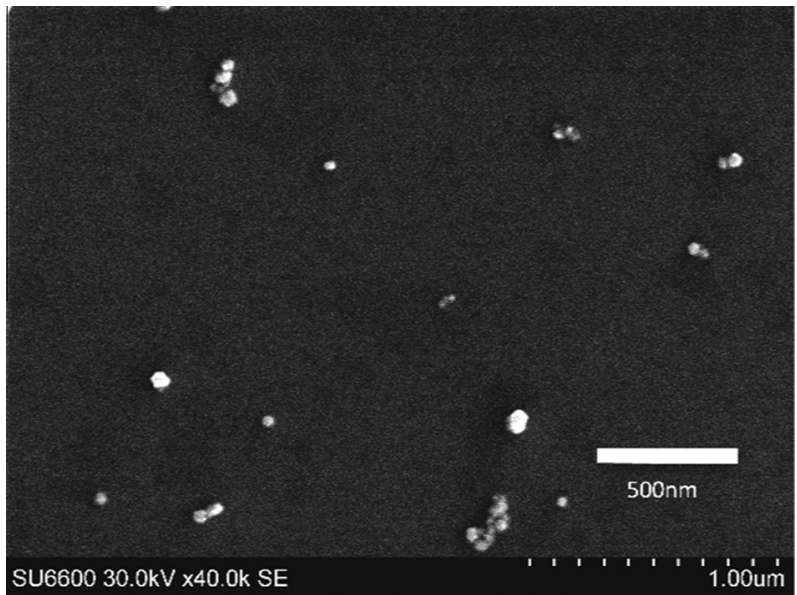

Fig. 4. SEM image at scale bar of $1 \mu \mathrm{m}$ showing purified in-house synthesised silver nanoparticles as purchased dispersed in ethanol to a concentration of $10 \mathrm{ppm}$ by sonication using Ultrasonic Processor tip. SEM images of various silver nanoparticles, all prepared by dispersion in ethanol followed by sonication. Samples were spin coated onto silicon wafers before being analysed in the SEM.

Table 2

Results of disk diffusion assay using various types of silver nanoparticles against $E$. coli. Data is expressed as mean \pm SD of three independent experiments.

\begin{tabular}{|c|c|c|c|c|c|c|c|}
\hline $\begin{array}{l}\text { Nanoparticle } \\
\text { concentration (ppm) }\end{array}$ & $\begin{array}{l}\text { In-house } \\
\text { impure }\end{array}$ & $\begin{array}{l}\text { In-house } \\
\text { purified }\end{array}$ & $\begin{array}{l}\text { In-house supernatant } \\
\text { from purification }\end{array}$ & $\begin{array}{l}\text { Sigma citrate } \\
\text { stabilised AgNP }\end{array}$ & $\begin{array}{l}\text { Supernatant from sigma } \\
\text { citrate stabilised AgNP }\end{array}$ & $\begin{array}{l}\text { Sigma PVP } \\
\text { stabilised AgNP }\end{array}$ & $\mathrm{AgNO}_{3}$ \\
\hline \multicolumn{8}{|c|}{ Diameter of zone of inhibition $(\mathrm{cm})$} \\
\hline 0 & 0 & 0 & - & 0 & - & 0 & 0 \\
\hline 10 & $0.4 \pm 0.34$ & 0 & - & 0 & - & 0 & 0 \\
\hline 20 & $0.68 \pm 0.1$ & 0 & - & 0 & - & 0 & 0 \\
\hline 30 & $0.76 \pm 0.2$ & 0 & - & 0 & - & 0 & 0 \\
\hline 40 & $0.88 \pm .027$ & $0.93 \pm 0.74$ & - & 0 & - & 0 & $0.56 \pm 0.05$ \\
\hline 50 & $0.94 \pm 0.22$ & 0 & - & 0 & - & 0 & $0.73 \pm 0.04$ \\
\hline 60 & $1.12 \pm 0.37$ & 0 & - & 0 & - & 0 & $0.83 \pm 0.12$ \\
\hline 70 & $1.13 \pm 0.36$ & $1.01 \pm 0.85$ & - & 0 & - & 0 & $0.99 \pm 0.08$ \\
\hline 80 & $1.26 \pm 0.28$ & $0.64 \pm 0.18$ & - & 0 & - & 0 & $1.13 \pm 0.03$ \\
\hline 90 & $1.2 \pm 0.36$ & $1.05 \pm 0.35$ & - & 0 & - & 0 & $1.05 \pm 0.08$ \\
\hline 100 & $1.42 \pm 0.23$ & $0.98 \pm 0.13$ & - & 0 & - & 0 & $1.19 \pm 0.08$ \\
\hline Supernatant & - & - & $1.23 \pm 0.13$ & - & 0 & - & - \\
\hline
\end{tabular}



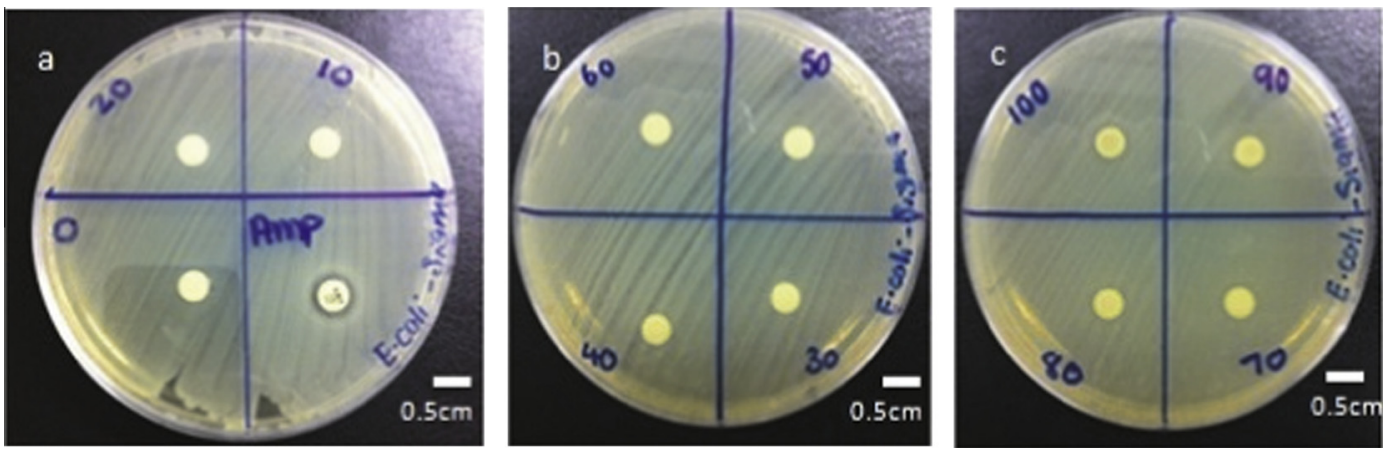

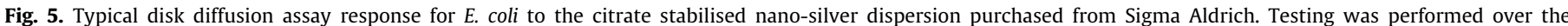

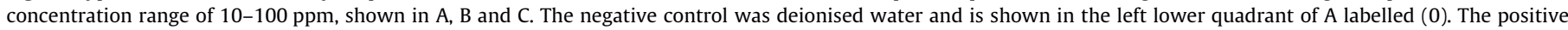
control was ampicillin and is shown in the right lower quadrant of A labelled (AMP).
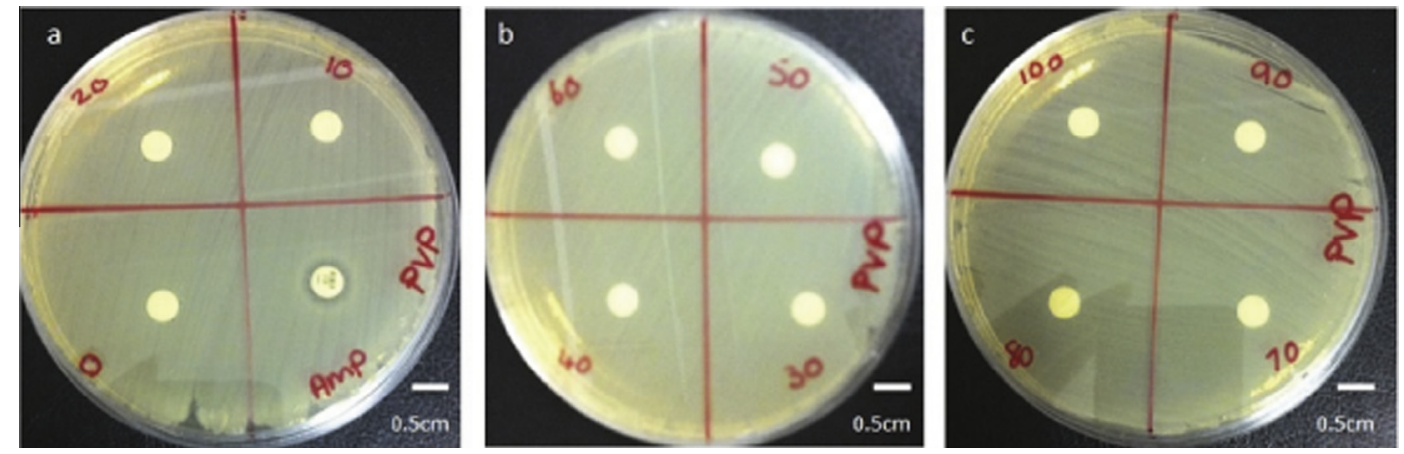

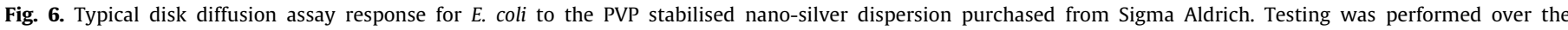

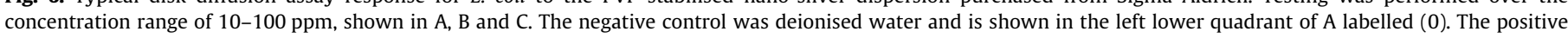
control was ampicillin and is shown in the right lower quadrant of A labelled (AMP).

Fig. 6 displays the disk diffusion assay carried out on E. coli using the commercial PVP stabilised nano-silver powder in concentrations ranging from $10 \mathrm{ppm}$ to $100 \mathrm{ppm}$. Similar to the citrate coated particles in Fig. 5, no inhibition zone can be identified around disks containing the nano-silver. This indicates that the disk diffusion does not provide any observable evidence for antimicrobial activity of PVP coated nano-silver towards E. coli, at concentrations as high as $100 \mathrm{ppm}$. The positive control, ampicillin, displayed a visible zone of inhibition.

These results somewhat contradict many literature reports indicating that nano-silver seemingly displays antimicrobial activity towards $E$. coli and other pathogenic bacteria [24,25,1]. A key difference however is that in these reports, the particles tend to be in-house synthesised particles which are subsequently stabilised. To mimic this, a further study was performed using nanoparticles synthesised as described by Fang et al. [8]. While the mechanism of action of nano-silver is still not fully understood, there is much evidence to suggest that the nanoparticle acts indirectly on the microorganism, acting as a vehicle for the active Ag+ ions which are continuously released from the surface of the particle [30] resulting in the antimicrobial effect. It is thus proposed here that stabilising surface coatings or capping agents such as PVP and citrate prevent or delay the rate at which ions are released from the surface of the nanoparticle, thereby suppressing any antimicrobial activity. In addition the omission of clean up or purification step in in-house nanoparticle syntheses may result in leftover starting material $\left(\mathrm{AgNO}_{3}\right)$ and ionic by-product both of which are known to be antimicrobial. To test this hypothesis, silver nanoparticles were synthesised as described previously, and tested both before and after purification procedure in order to compare their antimicrobial activity.
Fig. 7 shows the results of the disk diffusion assay on the inhouse synthesised nanoparticles without purification. There was a notable antimicrobial activity in the form of inhibition zones from particle concentrations of $30 \mathrm{ppm}$ upwards. Table 2 shows the diameters of each of the zones. There is a general trend of increasing zone diameter with increasing particle concentration, with the $30 \mathrm{ppm}$ particles showing an average inhibition zone of $0.76 \mathrm{~cm} \pm 0.2 \mathrm{~cm}$, and the $100 \mathrm{ppm}$ particles having an average inhibition zone of $1.4 \mathrm{~cm} \pm 0.2 \mathrm{~cm}$, which is greater than that observed for the ampicillin.

This result would seem to indicate a clear enhancement of the antimicrobial activity in the absence of any particle capping/coating agents. However before such a statement can be made it is crucial that the same study be performed for purified particles. Fig. 4 shows the activity of the in-house synthesised particles following a purification step to remove dissolved ions left over from the starting product. The nanoparticle solution was filtered using a $10 \mathrm{kDa}$ centrifugal filter unit by centrifuging at $2867 \times \mathrm{g}$ for $20 \mathrm{~min}$. The retentate was then re-suspended in ultrapure deionised water and brought forward for testing.

Fig. 8 shows the results of the assay carried out on the purified particles. It is evident that the purified particles are less antimicrobial than the impure particles in Fig. 3, with the largest average zone diameter being $1.05 \mathrm{~cm} \pm 0.35 \mathrm{~cm}$ compared with $1.42 \mathrm{~cm}$ for the impure particles. The supernatant from the clean up step was also assayed and this showed significant antimicrobial activity with an average inhibition zone diameter of $1.23 \mathrm{~cm} \pm 0.13 \mathrm{~cm}$. Atomic absorption spectroscopy was subsequently carried out on the supernatant and revealed a silver ion concentration of $31.08 \mathrm{ppm}$. It can therefore be concluded that dissolved ions and impurities present in the in-house synthesised particles are strongly antimicrobial. 

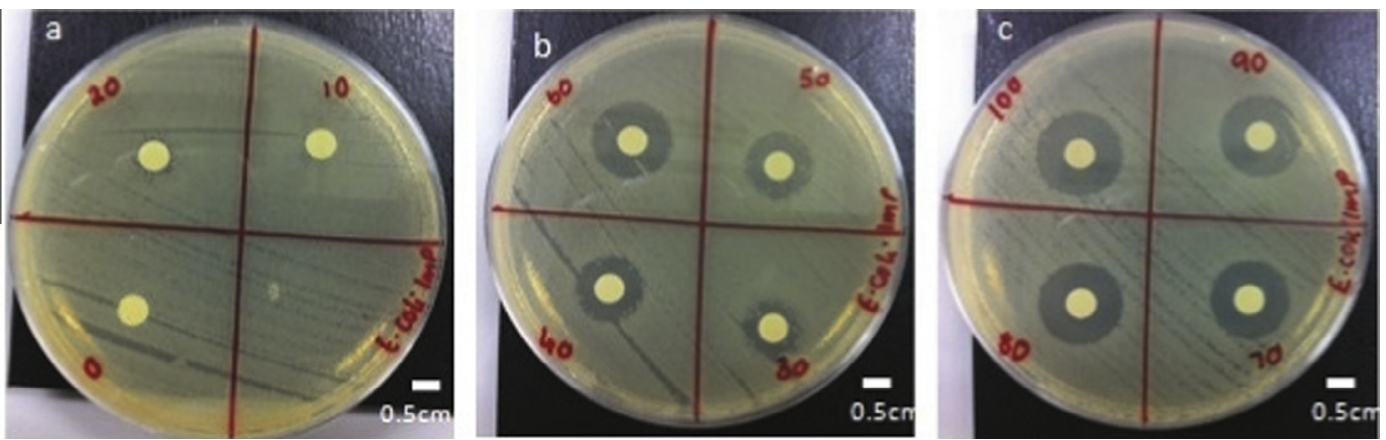

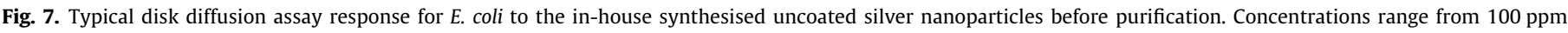
down to $10 \mathrm{ppm}$, shown in A, B and C. The negative control was deionised water and is shown in the left lower quadrant of A labelled (0).
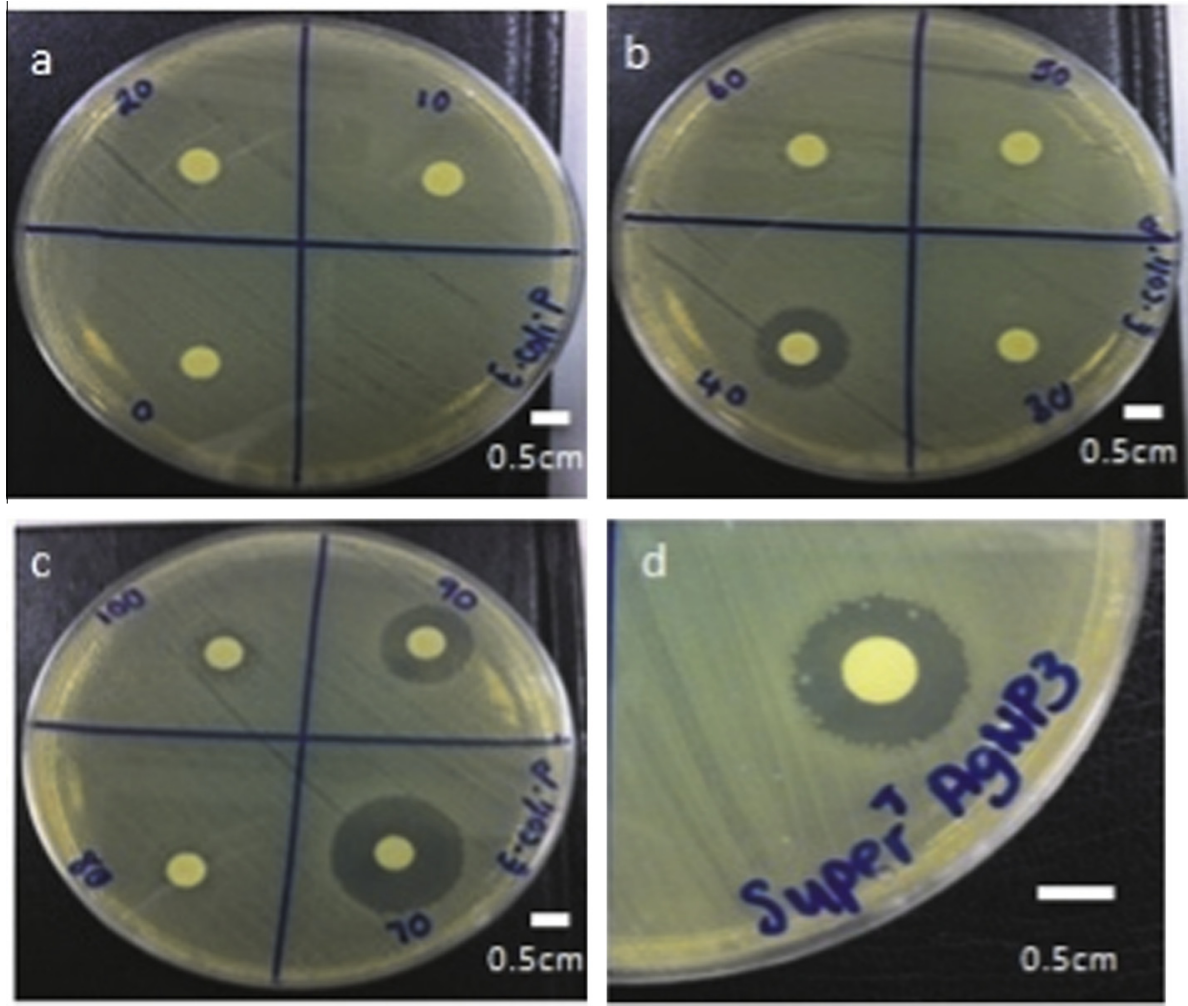

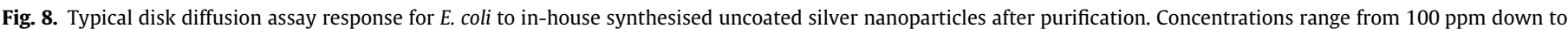

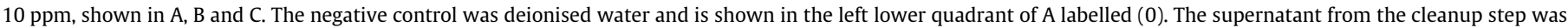
also assayed and shown in $\mathrm{d}$.

Fig. 8 therefore suggests that in the case of in-house synthesised particles, it is possible that there is a contribution to the antimicrobial activity due to impurities in the form of left over starting product in the sample. However, the uncoated particles do display antimicrobial activity over a range of concentrations, whereas the Sigma Aldrich ${ }^{\mathrm{TM}}$ citrate coated nanoparticles displayed no antimicrobial effect at these concentrations.

In order confirm the theory that antimicrobial activity of insufficiently cleaned nanoparticles is due to impurities, the starting product (silver nitrate) was also tested for antimicrobial activity. The results are shown in Fig. 9. It can be seen that inhibition zones are caused at concentrations of $40 \mathrm{ppm}$ upwards, with the $100 \mathrm{ppm}$ particles displaying an average zone diameter of $1.19 \mathrm{~cm} \pm 0.08 \mathrm{~cm}$. This is comparable to the results seen for the impure particles, which again would suggest that the activity seen by the impure particles may be mostly induced by remnant silver nitrate.
The supernatant from the purification step carried out on the Sigma Aldrich ${ }^{\mathrm{TM}}$ citrate coated particles was also tested for antimicrobial activity. According to the manufacturer, there should minimum ionic by-product in the supernatant. This was verified using atomic absorption spectroscopy, which revealed a maximum silver ion concentration of $1.48 \mathrm{ppm}$. This low concentration of ions is to be expected due to the fact that the citrate coated particles have been shown to display poor antimicrobial activity, due to their inefficiency at releasing ions. Fig. 10 shows that the supernatant of the Sigma Aldrich ${ }^{\mathrm{TM}}$ citrate coated nanoparticles does not have any antimicrobial activity due to the reduced level of ionic silver present in the sample. This is expected as the dispersion is of very high purity and it has already been observed that the citrate coated particles are not antimicrobial, due to the fact that they are not efficient at releasing ions. While the presence of silver species had previously been detected using AAS (detailed above), as a 

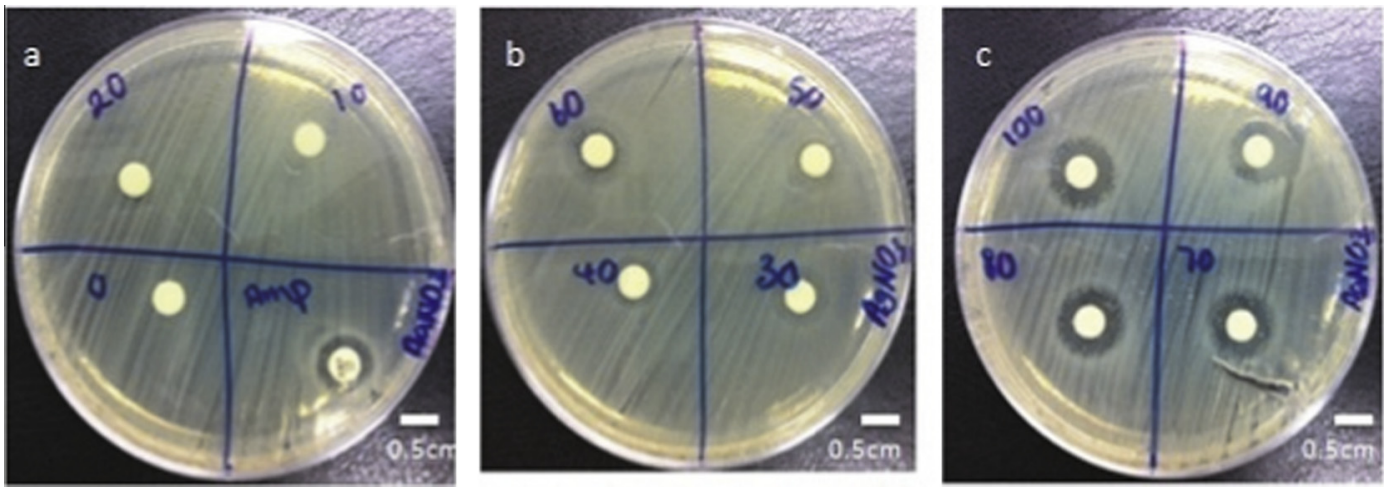

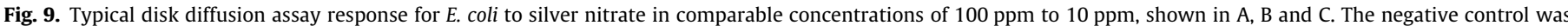

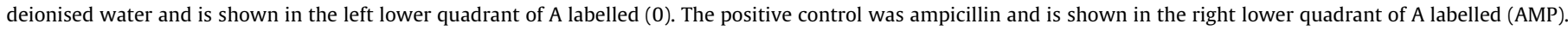

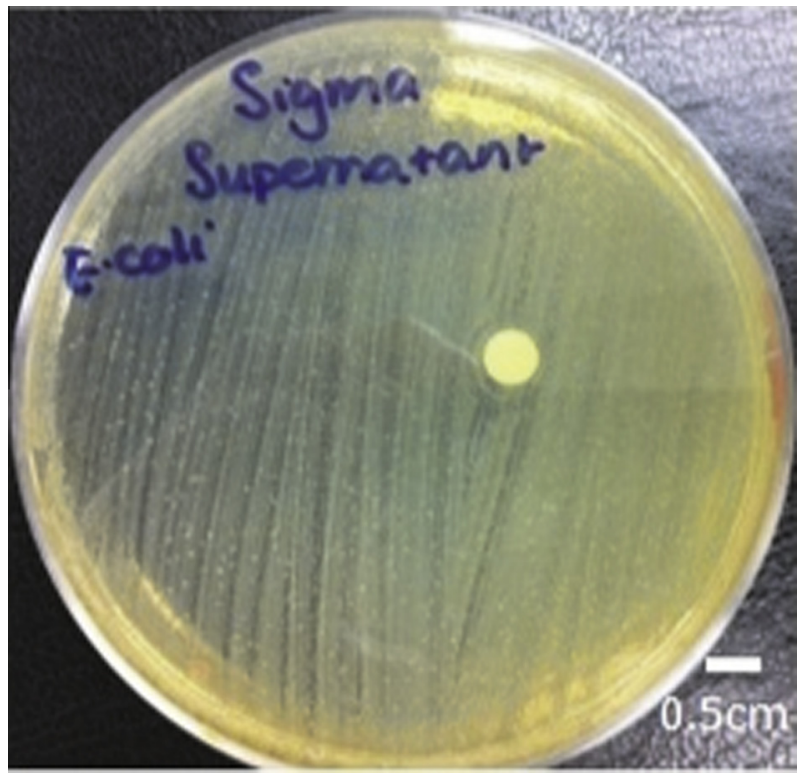

Fig. 10. Typical disk diffusion assay response for $E$. coli to the resultant supernatant from the purification process of the Sigma Aldrich ${ }^{\mathrm{TM}}$ citrate coated particles.

further test, the supernatants from both the Sigma Aldrich ${ }^{\mathrm{TM}}$ particles and the in-house synthesised particles were tested for the presence of silver ions by the addition of a chelating agent, $\mathrm{NaCl}$. The presence of silver ions in solution would result in the appearance of a cloudy precipitate on the addition of $\mathrm{NaCl}$ as the ions react with it to form silver chloride. It was noted that the addition of $\mathrm{NaCl}$ to the in-house nanoparticle supernatant resulted in the formation of a precipitate, indicating the presence of silver ions, while no precipitate was formed by the Sigma Aldrich ${ }^{\mathrm{TM}}$ citrate stabilised nanoparticle supernatant, providing confirmation of the absence of ions in the supernatant of the commercial silver nanoparticles.

Silver nanoparticles are thought to release ions from their surface, which is dependent on dissolved oxygen [14],[12]. In order to test the ability of silver nanoparticles to release ions in an agar based medium, the dissolved oxygen content of the nutrient agar was measured over the time it took for it to solidify. This was then compared to the dissolved oxygen content of nutrient broth which is used in the formulation of the agar.

Dissolved oxygen (DO) concentration was measured using a Vernier $^{\mathrm{TM}}$ Dissolved Oxygen Probe over a time period of 0-2500 s (to allow the agar to fully solidify). Results showed a marked difference in dissolved oxygen concentration between the broth (7 ppm DO) and agar (5 ppm DO) at the beginning of the experiment, while at $2500 \mathrm{~s}$, the level of dissolved oxygen in the agar had fallen to $3.5 \mathrm{ppm}$ while levels in the broth remained consistently in the range of 6 to $7 \mathrm{ppm}$. While more work would need to be carried out to ensure that changes in DO can cause an effect on ion production, it seems to indicate that carrying out antimicrobial activity assays in agar based media hinders the ability of silver nanoparticles to release ions and hence induce an antimicrobial effect.

\section{Discussion}

The promise of metal nanoparticles as new emerging antimicrobials is undoubtedly an exciting prospect, with significant benefits and commercial potential. Nano-silver in particular has been the focus of much attention with numerous consumer products claiming antimicrobial properties derived from nano-silver [29]. This is despite reports indicating that nano-silver has negligible antimicrobial properties and that it is the silver ion which is the source of the antimicrobial effect. Indeed the results presented in this paper have shown that the antimicrobial activity of silver nanoparticles can vary significantly depending on the physiochemical characteristics of the particle and that the these properties are dependent on the synthesis and processing of the nanomaterial prior to performing any antimicrobial assay. Three silver nanoparticles particles were assessed in this paper against E. coli; two commercially purchased and stabilised particles and one in-house synthesised particle. The in-house particle was tested in its asproduced state and in a purified state. The disk diffusion assay was chosen as it is the most common assay used in antimicrobial nanomaterial synthesis. The results indicated that neither of the commercial particles displayed any zones of inhibition with respect to $E$. coli. It should be noted that the manufacturer did not claim any antimicrobial properties of their particles. In contrast the in-house synthesised particle as-produced did display zones between $0.4 \mathrm{~cm}$ and $1.42 \mathrm{~cm}$ for the concentration range 10$100 \mathrm{ppm}$ respectively. However once the particle was purified, these zones were seen to decrease. The removed product from the purification step (supernatant) however exhibited comparable zones to the impure material. This supernatant product was shown to contain up to $31.08 \mathrm{ppm}$ of ionic silver which is a known to induce an antimicrobial effect. In addition residual un-reacted starting products such as silver nitrate are also expected to be present and itself can cause an antimicrobial effect. The results reported here highlight the need for an awareness of the nature of the material under test. This is very much in agreement with the findings Xiu et al. [30] who first reported observing no direct 
antimicrobial effects of nano-silver but rather attributed the effect to ionic silver. Despite this, there are still reports emerging which present the antimicrobial activity of silver nanoparticles with no regard to the potential presence of the ion or other impurities.

Sadeghi et al. [25] reported zones of inhibition of $2 \mathrm{~mm}$ for $S$. aureus and $1 \mathrm{~mm}$ for $E$. coli using silver nanoparticles synthesised by the borohydride reduction method at a concentration of around $5 \mathrm{ppm}$. However, no attempt to clean the particles after synthesis is evident [25]. Maneerung et al. [17] again used the disk diffusion assay to test nanoparticle-impregnated cellulose against $S$. aureus and $E$. coli. In this case, the samples used for the disk diffusion assay had been freeze dried, but all characterisation of the nanocomposite had been carried out before freeze drying. Hence it is not clear whether the freeze drying may affect the morphology of the particles, and/or remove ionic impurities from the sample. No antimicrobial assay before freeze drying was evident in the study [17].

The results presented here are not intended to contradict or undermine such reports but serve as a reminder of the importance of considering the physicochemical properties of the nanomaterial, a lesson which was learned by the nanotoxicology community in recent years, and applies to in-house synthesised particles predominantly. Such particles present challenges in determining the degree of coverage of coating or stabilising agents and may contain impurities in the form of ionic by-product and starting material.

In demonstrating the negligible particle specific antimicrobial behaviour of silver nanoparticles Xiu et al. [30] also found that silver nanoparticles which had been synthesised, stored and assessed anaerobically could not release ions on exposure to $E$. coli, verifying the active role of the ionic form of silver. The result indicated that oxidation of the particles caused release of silver ions, which increased the antimicrobial effect. In aerobic conditions several studies have suggested that the concentration of dissolved oxygen in solution has an impact on the dissolution rate of the ions from silver nanoparticles $[14,12]$. These studies suggest that dissolved oxygen in solution aids the surface oxidation of silver nanoparticles leading to ion release. Hence the higher the concentration of dissolved oxygen in solution, the higher the dissolution rate of the ions. This is reflected in the result presented in this paper where a decrease in the DO was identified in solidified agar in comparison to nutrient broth (3.5 ppm DO and 7 ppm DO respectively). As the mechanism of action of metallic nanoparticles is thought to be dependent on dissolved oxygen present in the surrounding media, it has been assumed that the antimicrobial activity observed in the agar for the disk diffusion assay is directly influenced by the local dissolved oxygen concentration. In addition, as the ionic species are released from the particle, their mobility would be reduced in agar as opposed to a liquid medium. It must be noted that the release of ions is not solely dependent on dissolved oxygen, but in the case of the commercial nano-silver studied here, the coating has a significant influence on the ability of the particle to release ions. Capping agents such as sodium citrate and PVP are often used in the synthesis of silver nanoparticles to create smaller and more stable particles. In theory the capping agent surrounds the surface of the particles and therefore it may hinder ion release. In a static test system such as agar, this effect could be even more pronounced.

These findings, combined with the findings of Xiu et al. therefore highlight an issue which underpins many of the obstacles faced in nanoparticle antimicrobial activity assays; the synthesis route by which the nanoparticle is produced, and subsequently the particle morphology and physiochemical characteristics can have a profound effect on the activity of that particle. This once again stresses the need for careful consideration of particle choice as well as the need for thorough physiochemical characterisation when evaluating antimicrobial activity. The consideration therefore should not be whether the assay method is applicable to nanomaterials but whether the particle itself is suitable for the assay. To determine the suitability of a particle for an assay and to allow for interlaboratory reproducibility, it is proposed here that as a precursor to any antimicrobial studies, a series of prioritised characterisation steps for the particle be considered. It is proposed that the following characterisation steps are key, based on the findings of this paper and those of Xiu et al. [30]:

- Confirmation of particle coatings or stabilising agents.

- Detailed breakdown of synthesis route and potential impurities.

- Size and shape characterisation in order to assess size dependent contributions.

- Presence of competing or complimentary process in the media, such as dissolved oxygen.

- The rate of ion dissolution and mobility for metallic nanoparticles.

In summary this study raises a number of issues about the antimicrobial assessment of nanoparticles, in particular with regard to material handling prior to performing an antimicrobial assessment. Ensuring that the nanoparticle is appropriately characterised will ensure reproducibility between nanoparticle batches and more importantly in inter-laboratory tests and for regulatory purposes. The later aspect is important when considered alongside the increasing number of nanoparticle based consumer products claiming to be antimicrobial. The increasing demand on research to demonstrate commercial relevance has led many material synthesis researchers to use basic antimicrobial studies to demonstrate a potential real-world application of newly synthesised particles and in the absence of standardised approaches, the transition from research laboratory to consumer products is becoming all too easy. In addition without truly understanding the nature of the material being assessed, the elucidation of any potential mechanism becomes impossible. It is hoped therefore that this report will open the debate on how best to approach and standardise the antimicrobial testing of nanomaterials.

\section{Conclusion}

This paper examined the appropriateness of the disk diffusion assay as an approach to assess the antimicrobial properties of nanoparticles, namely a selection of coated and uncoated nanosilver particles. The work demonstrated that for nanomaterials, the assay results are very much dependent on understanding the physicochemical characteristics of the nanoparticle and the potential antimicrobial mechanism of interaction. For the disk diffusion assay it was shown that coated and purified particles showed minimal antimicrobial activity. In comparison ionic silver present in the as-produced in-house synthesised particles showed zones of inhibition as large as $1.7 \mathrm{~cm}$. The result highlights that when selecting an assay it is crucially important to appropriately characterise the nanoparticle and ensure that the characterisation is fully integrated into the methodology of the assay. The relevance of the results in terms establishing protocols for method development for 'nanoparticle specific' antimicrobial properties was also discussed.

\section{Acknowledgments}

This work is funded by the Science foundation Ireland's Research Frontiers Programme and Fiosraigh Scholarship Programme. This work was conducted under the framework of the INSPIRE programme, funded by the Irish Government's Programme for Research 
in Third Level Institutions, Cycle 4, National Development Plan 2007-2013, supported by the European Union Structural Fund.

\section{References}

[1] A. Abdullah, Z. Hamid, J. Biol. Agric. Healthcare 3 (7) (2013) 67-69.

[2] N.V. Ayala-Núñez et al., Nanobiotechnol 5 (1-4) (2009) 2-9.

[3] A.W. Bauer, D.M. Perry, W.M. Kirby, AMA Arch Int. Med. 104 (2) (1959) 208 216.

[4] K.C. Bhainsa, S.F. D’Souza, Colloids Surf. B: Biointerfaces 47 (2) (2006) 160-164.

[5] D.K. Bhui, H. Bar, P. Sartar, G.P. Sahoo, S.P. De, A. Misra, J. Mol. Liq. 145 (1) (2009) 33-37. Branch, R.S. (2008). Image J.

[6] I. Chopra, J. Antimicrob. Chemother. 59 (2007) 587-590.

[7] A.J. Christy, M. Umadevi, Adv. Nat. Sci. Nanosci. Nanotechnol. 3 (2012) 1-4.

[8] J. Fang, C. Zhong, R. Mu, Phys. Lett. 401 (2005) 271-275.

[9] E. Guibal, S. Cambe, Bayle, J.M. Taulemesse, T. Vincent, J. Colloid Interface Sci. 393 (2013) 411-420.

[10] G. Guzmán, J. Dille, S. Godet, Int. J. Chem. Biomol. Eng. 2 (3) (2009) 104-111.

[11] H. Lara, N.V. Ayala-Nunez, L. Turrent, C.R. Padilla, World J. Microbiol. Biotechnol. 26 (4) (2010) 615-621.

[12] J. Liu, R.H. Hurt, Environ. Sci. Technol. Lett. 44 (6) (2010) 2169-2175.

[13] R. Lockyer, G.E. Hames, Analyst 84 (1959) 385-387.

[14] C.N. Lok, c.m. Ho, R. Chen, Q.Y. He, W.Y. Yu, H. Sun, P.K.H. Tam, J.F. Chiu, C.M. Che, J. Biol. Inorg. Chem. 12 (4) (2007) 527-534.

[15] Z. Lu, C. Mao, M. Meng, S. Liu, Y. Tian, L. Yu, B. Sun, M. Li, J. Colloid Interface Sci. 435 (2014) 8-14.
[16] Malvern: <http://www.malvern.com/labeng/technology/dynamic_light scattering/dynamic_light_scattering.htm>.

[17] T. Maneerung, S. Tokura, R. Rujiravanit, Carbohydr. Polym. 72 (1) (2008) $43-$ 51.

[18] D. Manikprabhu, K. Lingappa, J. Pharm. Res. 6 (2013) 255-260.

[19] R.C. Murdock, L. Braydich-Stolle, A.M. Schrand, J.J. Schlager, S.M. Hussain, Toxicol. Sci. 101 (2) (2008) 239-253.

[20] A. Nanda, M. Saravanan, Nanomedicine 5 (4) (2009) 452-456.

[21] R. Nayak, N. Pradhan, D. Behera, K.M. Pradhan, S. Mishra, L.B. Sukla, B.K. Mishra, J. Nanoparticle Res. 13 (2011) 3129-3137.

[22] OECD 2014 <http://www.oecd.org/science/nanosafety/45910212.pdf>.

[23] M. Rai, A. Yadav, A. Gade, Biotechnol. Adv. 27 (2009) 76-83.

[24] B. Sadeghi, M. Jamali, S.H. Kia, A. Amini Nia, S. Ghafari, Int. J. Nano Dim 1 (2) (2010) 119-124.

25] B. Sadeghi, F.S. Garmaroudi, M. Hashami, H.R. Nezad, A. Nasrollahi, S. Ardalan, Adv. Powder Technol. 23 (1) (2012) 22-25.

[26] A.R. Shahaverdi, A. Fakhimi, H.R. Shahverdi, S. Minaian, Nanomed.: Nanotechnol. Biol. Med. (2007) 168-171.

[27] I. Sondi, D.V. Goia, E. Matijevic, J. Colloid Interface Sci. 260 (1) (2003) 75-81.

[28] D.B. Warheit, Toxicol. Sci. 101 (2) (2008) 183-185.

[29] Woodrow Wilson: <http://www.nanotechproject.org/inventories/consumer/ browse/products/5107/>.

[30] Z.M. Xiu, Q.B. Zhang, H.K. Puppala, V.L. Colvin, J.J. Alvarez, Nano Lett. 12 (8) (2012) 4271-4275.

[31] N. Padmavathy, R. Vijayaraghavan, Sci. Technol. Adv. Mater. 9 (3) (2008) 1-7. 